Herzschr Elektrophys 2018 - 29:393-400 https://doi.org/10.1007/s00399-018-0597-4 Eingegangen: 8. August 2018

Angenommen: 10. September 2018

Online publiziert: 10. Oktober 2018

๑) Der/die Autor(en) 2018

CrossMark

Ester Herrmann - A. Ecke - S. Fichtlscherer · A. M. Zeiher · B. Assmus

Med. Klinik III, Kardiologie, Goethe Universität Frankfurt, Frankfurt, Deutschland

\title{
Pulmonalarterieller Drucksensor zur ambulanten Beurteilung des ventrikulären Füllungsdrucks bei fortgeschrittener Herzinsuffizienz
}

\section{Was ist bei der Nachsorge zu beachten?}

An chronischer Herzinsuffizienz leiden in Deutschland aktuell bis $\mathrm{zu} 3$ Millionen Menschen, und die Prävalenz steigt mit zunehmendem Alter. Somit ist die chronische Herzinsuffizienz eine der häufigsten Herz-Kreislauf-Erkrankungen und die häufigste Hauptdiagnose in deutschen Krankenhäusern. Jedes Jahr sterben rund 50.000 Menschen an den Folgen einer Herzinsuffizienz.

Die Krankheitskosten infolge einer Herzinsuffizienz betrugen im Jahr 2008 rund 3,2 Mrd.Euro. Etwa $45 \%$ sind bedingt durch die stationäre Krankenhausversorgung [1].

Beim Übergang von einem kompensierten Stadium bzw. stabiler Herzinsuffizienz hin zu einer Linksherzdekompensation treten in charakteristischer Reihenfolge pathophysiologische Veränderungen auf.Zuerst ist unabhängig von der Art des Linksherzversagens (Nachlasterhöhung, Pumpversagen etc.) ein Anstieg der linksatrialen und in der Folge auch der pulmonalarteriellen Druckwerte zu detektieren. Dies kann einer kardialen Dekompensation bis zu ca. 20 Tage vorausgehen. Im weiteren Verlauf kommt es zunächst zur autonomen Adaptation durch das Nervensystem, dann sinkt die thorakale Impedanz. Erst wenige Tage vor der Dekompensation treten die klassischen Symptome Gewichtszunahme und vermehrte Dyspnoe auf [2].

Zur Messung der linksseitigen Füllungsdrücke wird idealerweise eine Herz- katheteruntersuchung mit Bestimmung des linksventrikulären enddiastolischen Drucks (LVEDP) durchgeführt. Im ambulanten Umfeld kann bei Fehlen einer Mitralklappenstenose alternativ auch der linksatriale Druck verwendet werden, der jedoch bisher nur im Rahmen von klinischen Studien ambulant erfasst werden kann. Um eine arterielle Punktion zu vermeiden, kann bei der venösen Rechtsherzkatheteruntersuchung (Einschwemmkatheter) alternativ der pulmonalkapilläre Verschlussdruck (Wedge-Druck) anstelle des LVEDP verwendet werden, der bei Fehlen einer eigenständigen pulmonalarteriellen Hypertonie sehr gut mit dem diastolischen pulmonalarteriellen Druck korreliert [3].

Angesichts der Invasivität ist die Rechtsherzkatheter-basierte Messung der Füllungsdrücke jedoch reserviert für spezielle Szenarien (z.B. Vorbereitung oder Listung zur Herztransplantation, Evaluation für ein Assist-Device, Differenzialdiagnose Schock).

\section{Pulmonalarterieller Druck- sensor CardioMEMSTM _ CHAMPION-Studie}

In Anlehnung an die Rechtsherzkatheteruntersuchung wurde das CardioMEMS ${ }^{\mathrm{TM}}-\mathrm{HF}-S y s t e m$ (Abbott, Atlanta, GA, USA) entwickelt, welches die Messung der pulmonalarteriellen Drücke nichtinvasiv, drahtlos und täg- lich in ambulanten Eigenmessungen durch den Patienten ermöglicht. Bei CardioMEMS $^{\mathrm{TM}}$ handelt es sich um einen Sensor ohne eigene Stromversorgung (passives Implantat), der über eine Rechtsherzkatheteruntersuchung in eine Pulmonalarterie implantiert wird. Der Sensor besteht aus einer Spule und 2 Schleifen. Letztere gewährleisten, dass der Sensor die Arterie nicht verlegt und der Blutfluss nicht blockiert wird (- Abb. 1 und 2).

Der PAP-Sensor („pulmonary artery pressure sensor") entspricht einer in den Körper eingebrachten Stimmgabel, welche durch einen von außen eingebrachten Energiepuls in Schwingungen versetzt wird. Je nach Höhe des PAP schwingt die Spule unterschiedlich stark. Anhand der Frequenz des Echos kann in der Folge der Blutdruck in den Lungengefäßen ermittelt werden. Der Patient wird angeleitet, die täglichen ambulanten Druckmessungen über ein externes Messkissen, welches eine spezielle Antenne beinhaltet, selbstständig durchzuführen. Die Messung selbst dauert nur wenige Minuten, und unmittelbar im Anschluss erfolgt die Übertragung durch das elektronische System des Patienten an eine sichere Website, auf die der behandelnde Arzt Zugriff hat. Anhand der Druckmesswerte kann der Arzt Behandlungsentscheidungen treffen und mit dem Patienten frühzeitig Modifikationen der Medikation entsprechend der 


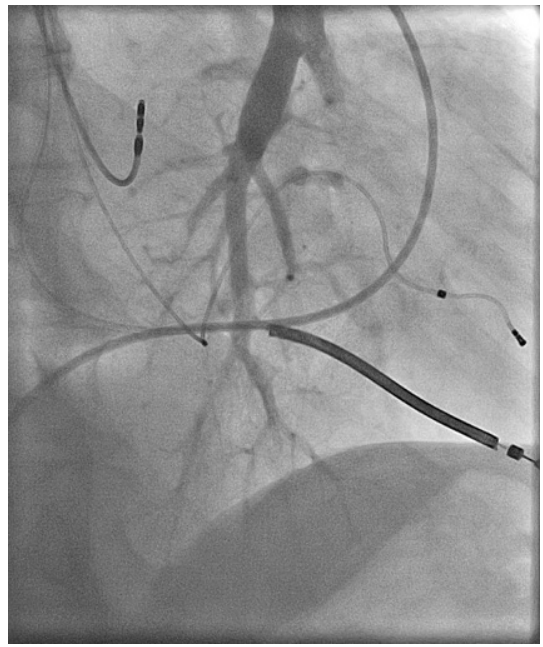

Abb. 1 ॥ Pulmonalisangiographie. (Abdruck mit freundl. Genehmigung der Firma Abbott)

gemessenen pulmonalarteriellen Druckwerte besprechen.

Die Implantation selbst kann als sicher bezeichnet werden. In der CHAMPION-Zulassungsstudie kam es bei 550 Patienten zu 8 (1\%) Device- oder System-assoziierten Komplikationen in den ersten 6 Monaten sowie zu 7 (1\%) Interventions-assoziierten unerwünschten Ereignissen [4]. Daten der ersten 2000 kommerziell implantierten Sensoren aus den USA zeigen eine vergleichbar geringe Komplikationsrate.

In der CHAMPION-Studie wurden 550 Patienten mit chronischer Herzinsuffizienz NYHA III und vorangegangener Hospitalisierung aufgrund kardialer Dekompensation innerhalb der letzten 12 Monate mit einem CardioMEMS ${ }^{\mathrm{TM}_{-}}$ Sensor versorgt. In der Interventionsgruppe $(n=280)$ wurden tägliche ambulante Eigenmessungen des pulmonalarteriellen Drucks durch den Patienten durchgeführt. Das Herzinsuffizienzmanagement mit entsprechenden Therapiemodifikationen erfolgte anhand Klinik und hämodynamischer Parameter, während in der Kontrollgruppe $(n=270)$ die PAP-Werte des Patienten dem Studienarzt nicht übermittelt wurden, um die Therapie zu titrieren. Der primäre Endpunkt, die Anzahl an Hospitalisierungen aufgrund von kardialer Dekompensation nach 6 Monaten, zeigte eine signifikante Reduktion in der Interventionsgruppe im Vergleich zur Kontrollgruppe [5]. Durch das PAP-gesteuertes Herzinsuffi-

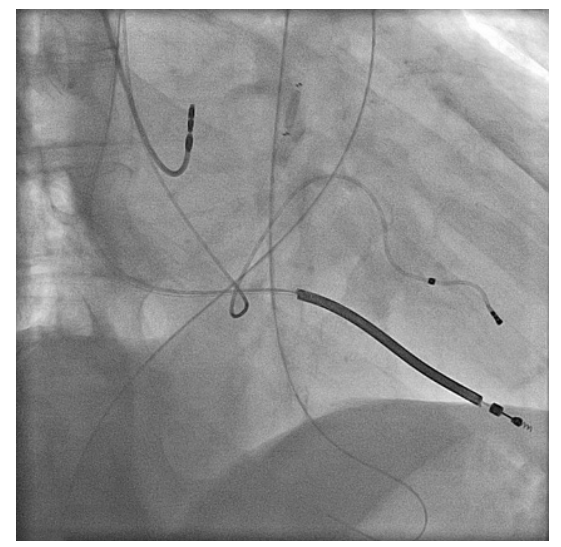

Abb. $2 \Delta$ CardioMEMS ${ }^{\text {TM}}$-Implantation in die zuvor dargestellte Pulmonalarterie. (Abdruck mit freundl. Genehmigung der Firma Abbott)

zienzmanagement zeigte sich über den mittleren Beobachtungszeitraum von 15 Monaten die Zahl der Krankenhauseinweisungen wegen progredienter Herzinsuffizienz noch weiter um $37 \%$ reduziert, begleitet von einer signifikant verbesserten Lebensqualität sowie signifikant reduzierten pulmonalarteriellen Druckwerten [4]. Wichtig ist auch, dass durch die Telemonitoring-getriggerte intensivierte diuretische und Herzinsuffizienztherapie keine Verschlechterung der Nierenfunktion gesehen wurde. Zudem wurde im Mittel eine höhere Dosis von ACE-Hemmer und $\beta$-Blocker erreicht [6]. Im Jahr 2016 wurde das LangzeitFollow-up der Patienten publiziert [4]. Hier wurde für den weiteren Verlauf bei den Patienten der ursprünglichen Kontrollgruppe das Herzinsuffizienzmanagement geändert hin $\mathrm{zu}$ einem PA-Druck-basierten Herzinsuffizienzmanagement. Durch dieses Cross-overManöver gelang es, die jährliche Hospitalisierungsrate in der Kontrollgruppe auf das Niveau der ursprünglichen Interventionsgruppe zu senken.

Auf Basis der randomisierten Studie wurde das CardioMEMS ${ }^{\mathrm{TM}}$-System in den USA und Europa zugelassen. Analysen der ersten 2000 Patienten, die das System kommerziell erhalten haben, zeigen, dass es auch im täglichen Leben neben einer anhaltend hohen Therapieakzeptanz durch den Patienten (>90\% tägliche Messungen nach 1 Jahr) $\mathrm{zu}$ einem starken Abfall der pulmo- nalarteriellen Druckwerte im Verlauf kommt, unabhängig von der AusgangsPumpfunktion. Zudem wurde kürzlich dem ACC eine gematchte Fall-KontrollStudie vorgelegt mit >1000 Patienten. Hier findet sich eine statistisch hochsignifikante Reduktion der Mortalität durch das PA-Druck-gesteuerte Herzinsuffizienzmanagement.

Aufgrund der Neuheit der Technologie gibt es in der Literatur bisher noch keine Untersuchungen zur erforderlichen Qualitätssicherung, und es fehlen Daten aus dem deutschen Gesundheitssystem.

Zudem stellt die alleinige Messung der PA-Druckwerte lediglich eine Diagnostik dar (vergleichbar der Blutzuckermessung), so dass die therapeutischen Konsequenzen in Abhängigkeit von den gemessenen Werten individuell zu überprüfen sind. Daher ist es von großer Bedeutung, die Nachsorge zu standardisieren und parallel zu evaluieren, weil nur durch eine korrekte Interpretation der Messwerte und angemessene Therapieanpassung die in den Studien beobachteten positiven Effekte in den Alltag übertragbar sind. Auch ist der langfristige Personal- und apparative Aufwand für dauerhaftes Telemonitoring sowie die langfristige Akzeptanz bei den Patienten derzeit noch unklar. In Europa erfolgt u. a. eine Erfassung des Zeitaufwands für PA-Druck-gesteuertes Herzinsuffizienzmanagement gegenwärtig durch das MEMS-HF-Register, welches primär die Sicherheit und Durchführbarkeit des hämodynamischen Telemonitorings in Deutschland, Irland und den Niederlanden untersucht.

\section{„Standard operating procedure" für PAP-Monitoring}

Grundsätzlich besteht für die Implantation des CardioMEMS ${ }^{\mathrm{TM}_{-}}$-Systems nach den ESC-Leitlinien 2016 eine Klasse-IIbEmpfehlung für Patienten mit symptomatischer Herzinsuffizienz nach stattgehabter Hospitalisierung, um das Risiko für weitere Hospitalisierungen $\mathrm{zu}$ reduzieren. Zudem sollte die Herzinsuffizienztherapie so weit wie möglich bereits optimiert sein.

Nach ambulanter und stationärer Identifikation potenzieller Patienten erfolgt an unserem Zentrum bereits vor 
Implantation eine ausführliche Patientenschulung zur Herzinsuffizienz (Basisüberwachung, Medikamente). Am Tag nach der Sensorimplantation erfolgen stationär durch die Heart Failure Nurses eine weitere ausführliche Herzinsuffizienzschulung sowie die Einweisung in die PAP-Sensorabfrage. Erst nachdem der Patient mindestens einmal eigenständig erfolgreich eine Messung durchgeführt hat, wird er nach Hause entlassen. Für die ambulante Nachsorge hat sich ein Team aus 2 Heart Failure Nurses und 3 Herzinsuffizienzärzten etabliert. Während die Routine-Nachsorge werktäglich grundsätzlich durch die Nurse (regelmäßige Telefonkontakte mit Abarbeiten eines standardisierten Fragebogens) erfolgt, sind wochentags für Rückfragen (Therapieanpassungen) und an den Wochenenden und Feiertagen wechselnd nach Dienstplan die Ärzte verantwortlich. Die Autoren schätzen aufgrund eigener Erfahrungen, dass für 50 Hämodynamik-Telemonitoring-Patienten eine $50 \%$ Heart-Failure-Nurse-Stelle erforderlich ist sowie $20 \%$ einer Arztdienststelle. Im Folgenden werden Inhalte der „standard operating procedure" (SOP) wiedergegeben, welche die Autoren mit dem Ziel einer standardisierten Nachsorge für das hämodynamische Monitoring und PA-Druck-gesteuerte Herzinsuffizienzmanagement von Patienten im Stadium NYHA III entwickelt haben. Die SOP gibt Handlungsempfehlungen für die Herzinsuffizienzärzte und die Heart Failure Nurses, die für die Betreuung von Patienten mit PAP-Sensor am interdisziplinären Frankfurter Herzinsuffizienzzentrum verantwortlich sind. Alle nachfolgend aufgeführten Therapieempfehlungen der SOP basieren auf subjektiven Einschätzungen und Erfahrungswerten der Autoren und sind nicht durch klinische Evidenz abgesichert.

Grundsätzlich sind folgende primäre pulmonalarterielle Druckwerte für alle Patienten anzustreben:

- PAP systolisch: $15-35 \mathrm{~mm} \mathrm{Hg}$

- PAP diastolisch: 8-20 mm Hg

- Mittlerer PAP: 10-25 mm Hg

Diese Werte müssen anhand der individuellen Gegebenheiten jedoch häufig bereits initial und im Verlauf mehrfach

Herzschr Elektrophys 2018 - 29:393-400 https://doi.org/10.1007/s00399-018-0597-4

(c) Der/die Autor(en) 2018

E. Herrmann · A. Ecke · S. Fichtlscherer · A. M. Zeiher · B. Assmus

Pulmonalarterieller Drucksensor zur ambulanten Beurteilung des ventrikulären Füllungsdrucks bei fortgeschrittener Herzinsuffizienz. Was ist bei der Nachsorge zu beachten?

Zusammenfassung

Hintergrund. Patienten mit fortgeschrittener Herzinsuffizienz leiden unter wiederholten Hospitalisierungen. Nichtinvasives hämodynamisches Telemonitoring zur Bestimmung des pulmonalvaskulären Füllungsdrucks kann Hospitalisierungen reduzieren. In diesem Beitrag wird über mögliche Kontrollintervalle und Standardisierung in der Nachsorge des hämodynamischen Telemonitorings berichtet.

Methoden. Eine Literaturrecherche sowie eigene Erfahrungswerte in der Nachsorge bezüglich des implantierbaren pulmonalarteriellen Drucksensors zum nichtinvasiven hämodynamischen Telemonitoring bei Patienten mit fortgeschrittener Herzinsuffizienz werden präsentiert.

Ergebnisse. Eine standardisierte Nachsorge für Patienten mit hämodynamischem Monitoring erfordert ein spezialisiertes Team aus Heart Failure Nurse und Herzinsuffizienzarzt.
Dieses Team arbeitet idealerweise nach einer einheitlichen SOP (, standard operating procedure ${ }^{\prime \prime}$ ), um für alle Patienten sowohl identische Kontrollintervalle als auch standardisierte Therapien bei klassischen hämodynamischen Veränderungen umzusetzen. Grundsätzlich müssen alle Therapiemodifikationen ärztlich angeordnet und bei Bedarf individuell modifiziert werden.

Schlussfolgerung. Die optimierte Nachsorge bei hämodynamisch geführtem Herzinsuffizienzmanagement erfordert neue Strukturen im deutschen Gesundheitssystem, damit der in Studien nachweisbare klinische Nutzen auch in der klinischen Routine umgesetzt werden kann.

Schlüsselwörter

Pulmonalarterieller Druck · Telemonitoring · Hämodynamik · Heart Failure Nurse . Qualitätskontrolle

\section{Pulmonary artery pressure sensor for ambulatory assessment of ventricular filling pressure in advanced heart failure. What should be considered for the follow-up care?}

\section{Abstract}

Background. Patients with advanced heart failure suffer from frequent hospitalizations. Noninvasive hemodynamic telemonitoring for assessment of pulmonary filling pressure has been shown to reduce hospitalizations. In this article, our experience with possible control intervals and the standardization of the follow-up care of hemodynamic telemonitoring is reported.

Methods. A literature search and our own experience in the follow-up care concerning the implantable pulmonary artery pressure sensor for noninvasive hemodynamic telemonitoring in patients with advanced heart failure are presented.

Results. For standardized follow-up care of heart failure patients with hemodynamic monitoring a specialized team consisting of a heart failure nurse and heart failure physi- cian is essential. These teams should ideally work based on a unique standard operating procedure (SOP) to ensure standardized control intervals and a standardized approach to classical hemodynamic changes. However, all therapeutic recommendations have to be prescribed by a physician and must be modified if individually appropriate.

Conclusion. Optimized follow-up care for hemodynamically guided heart failure management requires the implementation of novel structures in the German health care system in order to transfer the clinical benefit from clinical trials into daily routine.

\section{Keywords}

Pulmonary artery pressure - Telemonitoring . Hemodynamic monitoring $\cdot$ Heart failure nurse - Quality control 


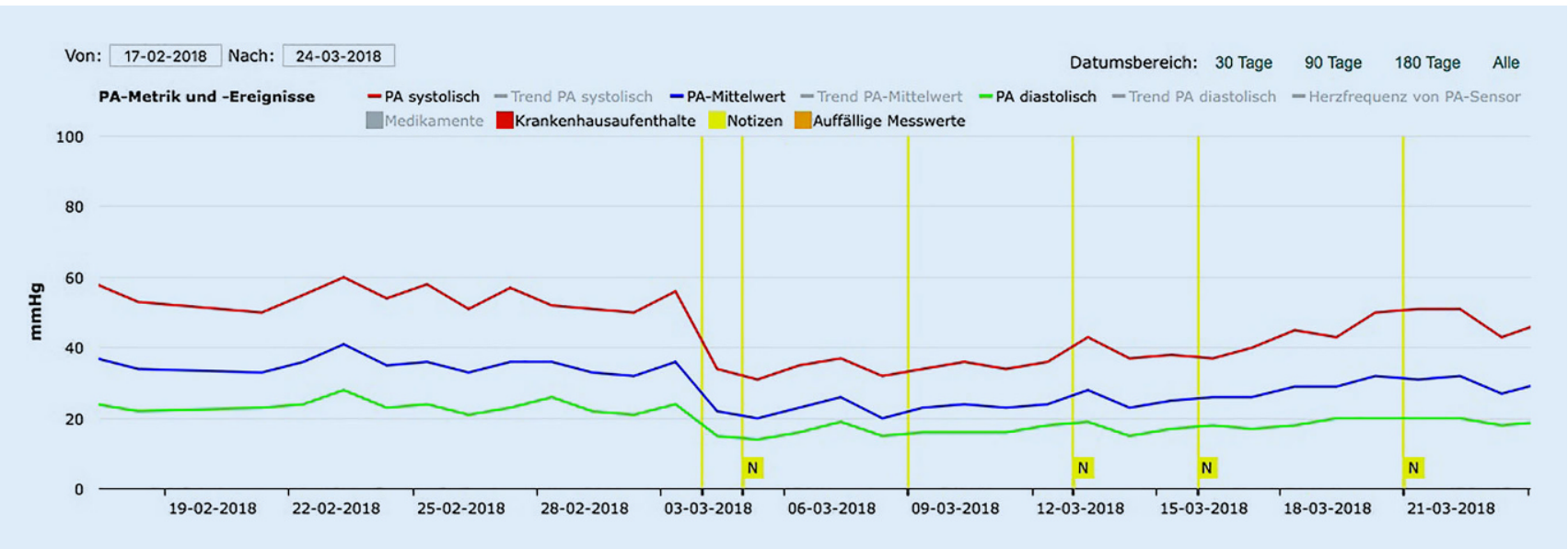

Abb. 3 ॥ Symptomatische Hypovolämie im Rahmen eines pulmonalen Influenza-Infekts mit frühzeitigem Erkennen eines PA-Druckabfalls mittels Telemonitoring. PA-Druckkurven in $\mathrm{mm} \mathrm{Hg}$ (rot systolischer, blau mittlerer, grün diastolischer PADruck) dargestellt über die Zeit. Gelbe vertikale Balken Telefonkontakte mit zunächst Pausieren von Torasemid, Amlodipin und Ramipril sowie vermehrter Flüssigkeitsaufnahme; im Verlauf nach Besserung des klinischen Zustands und der Vitalparameter allmähliches Einschleichen der Medikamente möglich

modifiziert werden. Letztlich werden durch Veränderungen der pulmonalarteriellen Druckwerte Veränderungen des Volumenstatus erfasst, die entsprechende therapeutische Anpassungen triggern und unterschiedliche Betreuungsintervalle erfordern.

\section{Euvolämie}

Die Euvolämie ist definiert als Zustand, bei dem der Patient optimal rekompensiert ist ohne Zeichen der Minderperfusion. Dies kann individuell bei stark unterschiedlichen pulmonalarteriellen Druckwerten der Fall sein, in Abhängigkeit von der Dauer der Grunderkrankung und einem möglicherweise sekundären Anstieg der pulmonalvaskulären Widerstände. Wenn die PA-Druckwerte trotz Ausschöpfung aller medikamentösen Therapieoptionen hoch bleiben und der Patient symptomatisch ist, empfehlen die Autoren häufig eine weitere Diagnostik (falls noch nicht erfolgt), z.B. mittels transösophagealer Echokardiographie zum Ausschluss begleitender Klappenvitien und/oder die Reevaluation mittels Rechtsherzkatheteruntersuchung und Vasoreaktivitätstestung. Sollten trotz aller Bemühungen die PA-Druckwerte dennoch hoch bleiben, so müssen letztlich die patientenindividuellen Ziel- und Grenzwerte ("threshold settings") angepasst werden. Auch wenn der Patient normale PA-Druckwerte nicht toleriert (z. B. Verschlechterung der Nierenfunktion, Hypotonie, Schwindel), sind die Referenzwerte von den Herzinsuffizienzärzten individuell an den Patienten anzupassen, und die individuell definierten euvolämen Werte sind zu verwenden.

Eigene Erfahrungen haben gezeigt, dass die im Alltag gemessenen Druckwerte unter regulärer körperlicher Aktivität häufig höher sind als die initial im Herzkatheterlabor gemessenen Druckwerte. Somit versuchen die Autoren zunächst bei allen Patienten, durch Intensivierung der Herzinsuffizienztherapie eine Reduktion bzw. Normalisierung des diastolischen pulmonalarteriellen Drucks zu erreichen, ohne dass sich die Nierenfunktion verschlechtert bzw. die Patienten hypotensiv werden. Gewöhnungsbedürftig für alle Beteiligten ist die isolierte Behandlung der Druckwerte, ohne dass die Patienten über neue und vermehrte Beschwerden berichten.

Hintergrund der PA-Druck-gesteuerten Therapieanpassung ist neben der $\mathrm{Da}$ ten der CHAMPION-Studie auch die Arbeit von Zile et al. [7], die zeigen konnte, dass bereits eine diastolische Drucksenkung von $5 \mathrm{~mm} \mathrm{Hg}$ mit einer $30 \%$ igen Reduktion des Mortalitätsrisikos assoziiert ist.

In unserer Kohorte von langjährig schwer herzinsuffizienten Patienten mit bereits vor CardioMEMS ${ }^{\mathrm{TM}}$-Implantati- on medikamentös maximal auftitrierter Therapie gelang oft nur eine geringfügige diastolische PA-Drucksenkung, wobei trotzdem die Linksherzinsuffizienz-getriggerten Hospitalisierungen im Vergleich zur Zeit vor der CardioMEMS ${ }^{\mathrm{TM}}$ Implantation in den ersten 24 Monaten um mehr als $50 \%$ reduziert werden konnten.

Grundsätzlich wird das Telemonitoring von den Patienten und kooperierenden Hausärzten bisher sehr gut angenommen. Nach Implantation werden die Hausärzte kontaktiert und über das System und Programm informiert. Im weiteren Verlauf erhalten die betreuenden Hausärzte regelmäßige Berichte über die gemessenen pulmonalarteriellen Druckwerte ihrer Patienten und führen bei Bedarf Laborkontrollen (Nierenwerte) durch.

\section{Hypervolämie}

Eine akute Erhöhung der Druckwerte über die Referenzwerte hinaus gilt als Volumenüberladung. Dies tritt häufig auf, ohne dass vom Patienten entsprechende Symptome der kardialen Dekompensation wie Dyspnoe und Beinödeme berichtet werden. Eine Erhöhung der PAPWerte oberhalb des euvolämen Bereichs kann entweder kurzfristig (innerhalb von 24-72 h) mit einem steilen Anstieg in der täglichen Einzelmessung oder aber 


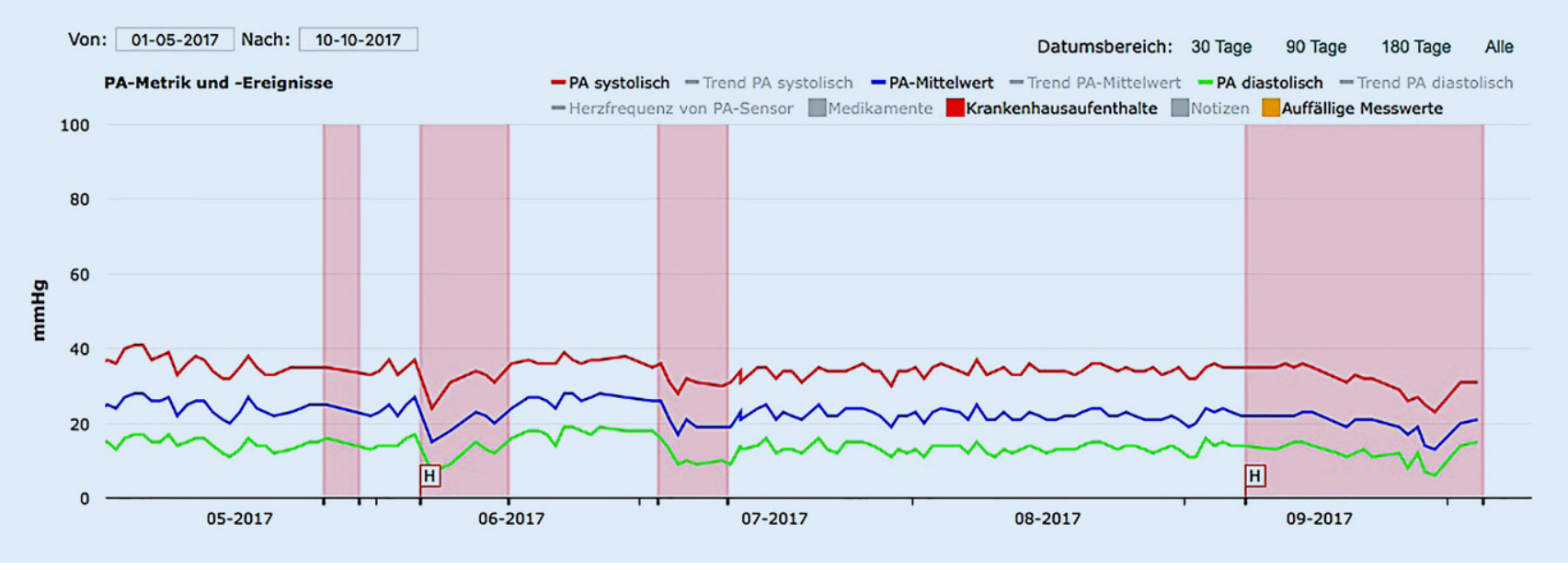

Abb. 4 ム Rezidivierende rechtsführende kardiale Dekompensationen mit akut auf chronischem Nierenversagen. Kaum PADruckveränderungen mittels Telemonitoring. PA-Druckkurven in $\mathrm{mm} \mathrm{Hg}$ (rot systolischer, blau mittlerer, grün diastolischer PA Druck) dargestellt über die Zeit. Rote vertikale Balken Hospitalisierungen. Kardiale Rekompensation mittels i. v.-Diuretika und inhalativ llomedin ${ }^{\circledR}$ als „, off-label use“. In Anbetracht des Allgemeinzustands des Patienten und in Rücksprache mit dem Patienten und den Angehörigen erfolgte im September 2017 die Deaktivierung des CRT-D-Aggregats und die Verlegung des Patienten auf eine Palliativstation

im Verlauf über mehrere Tage oder Wochen mit einem eher langsamen graduellen Anstieg auftreten.

Zur kurzfristigen Behandlung des diastolischen PA-Druckanstiegs sind entsprechend den begleitenden Blutdruckwerten entweder Diuretika oder Vasodilatanzien anwendbar, wobei parallel auf eine optimal ausdosierte Herzinsuffizienztherapie (ARNI oder ACEHemmer/AT1-Blocker, $\beta$-Blocker, Aldosteronantagonisten) $\mathrm{zu}$ achten ist. In der eigenen klinischen Praxis werden zur Behandlung von relevanten diastolischen PA-Druckanstiegen überwiegend Diuretika verwendet. Je nach individueller Begleittherapie wird ein Schleifendiuretikum ergänzt bzw. erhöht, oder es wird eine sequenzielle Nephronblockade vorgenommen, zum Teil auch nur passager. Die Autoren haben sehr gute Erfahrungen mit einer kurzzeitigen, über 2 bis 3 Tage anhaltenden Verdopplung der Diuretikaausgangsdosis gemacht. Meist kann schon am Folgetag nach der Therapieanpassung ein Abfall der pulmonalarteriellen Druckwerte festgestellt werden. Nach eigenen Erfahrungen kann durch die nur kurzfristige intensivierte Therapie eine anhaltende Verschlechterung der Nierenfunktion meist verhindert werden.

Aus diesem Vorgehen ist auch ersichtlich, dass v.a. geistig fitte Patienten für das Verfahren in Betracht kommen, denn die Patienten müssen in der Lage sein, auf telefonische Anordnung die richtigen Medikamente in der richtigen Dosierung über die entsprechende Zeit anzupassen. Bei anhaltend hohen PA-Druckwerten (fragliche Diuretikaresistenz) werden die Patienten um umgehende ambulante Vorstellung in der Herzinsuffizienzsprechstunde gebeten, um über eine tagesstationäre i.v.-Therapie oder eine stationäre Aufnahme entscheiden zu können.

Nach Veränderung der medikamentösen Therapie muss der pulmonalarterielle Druck engmaschig, d.h. mindestens 2- bis 3-mal wöchentlich, überprüft werden. Zudem werden erneute Patientenschulungen zur Herzinsuffizienz mit Schwerpunkt zur Salz- und Volumenrestriktion durchgeführt.

Wenn der Patient über die kardiale Dekompensation hinaus außerdem Hinweise für eine reduzierte Perfusion aufweist (Frage nach Blutdruck, kalten Extremitäten, Urinproduktion), wird eine umgehende Vorstellung zur stationären Aufnahme empfohlen.

Analog zum Vorgehen in der CHAMPION-Studie wird in der SOP der Autoren eine Anpassung der Diuretika (und/ oder Vasodilatatoren) empfohlen mit dem Ziel der PA-Drucksenkung, bis einer der folgenden Befunde eintrifft:
1. Serum-Kreatinin-Erhöhung um $20 \%$

2. Abfall des systolischen Blutdrucks um $20 \mathrm{~mm} \mathrm{Hg}$ bei Wechsel von Rückenlage in eine aufrechte Position 3. Symptomatische, systemische Hypotension, Orthostase

\section{Hypovolämie}

Bei Hypovolämie zeigt sich eine Verminderung der PAP-Werte unterhalb des als Euvolämie definierten Bereichs. Neben Orthostase kann es zur Verschlechterung der Nierenfunktion sowie allgemein der Perfusion kommen, ohne dass Symptome der Dekompensation auftreten. Die Verminderung der PAP-Werte unterhalb des euvolämen Bereichs kann entweder in der täglichen Einzelmessung oder aber im Verlauf über die Woche ersichtlich werden.

Behandlungsoptionen bestehen in der Reduktion oder im Pausieren von Diuretika. Darüber hinaus ist eine passager erhöhte Trinkmenge möglich. Bei posturaler Hypotension, insbesondere in sitzender Position und in Rückenlage, sollten Vasodilatatoren und/oder orale Nitrate vermindert oder pausiert werden. Wenn sich die Nierenfunktion verschlechtert, insbesondere bei Hypotension, sollten ACE-Hemmer/ARB/ ARNI/MRA-Dosierungen vermindert oder pausiert werden (Beispiel: • Abb. 3). 


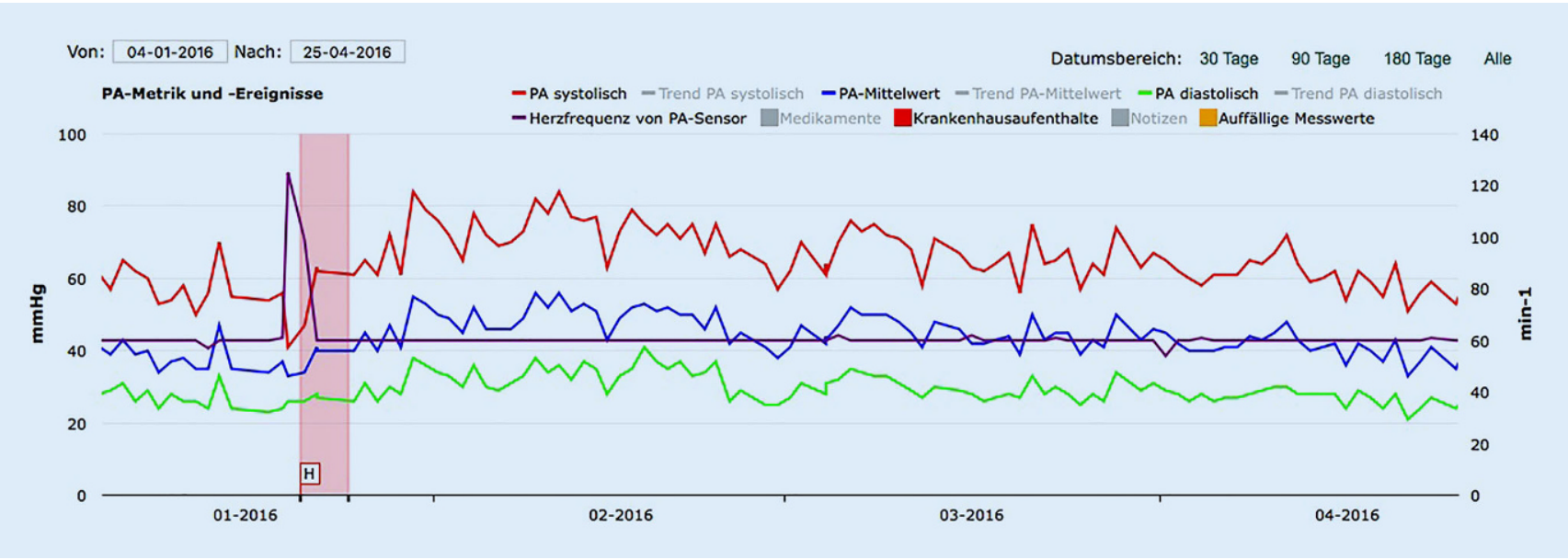

Abb. 5 ム Verhinderte kardiale Dekompensation durch frühzeitige Erkennung eines PA-Druckanstiegs aufgrund subjektiv nicht empfundenem, tachykard übergeleitetem Vorhofflimmern mittels Telemonitoring. PA-Druckkurven in mm Hg (rot systolischer, blau mittlerer, grün diastolischer PA-Druck) dargestellt über die Zeit. Violette Kurve Herzfrequenz vom PA-Sensor in $\mathrm{min}^{-1}$, roter vertikaler Balken Hospitalisierung aufgrund von tachykard übergeleitetem Vorhofflimmern, und elektrische Kardioversion, im Anschluss Amiodarontherapie

Wenn der Patient begleitend anhaltend hypoton ist sowie Zeichen und Symptome einer reduzierten Perfusion aufweist, wird eine stationäre Aufnahme und, je nach klinischer Erfordernis, eine vorsichtige i.v.-Volumengabe, Monitorüberwachung und/oder eine inotrope Therapie empfohlen.

\section{Dekompensation bei rechtsventrikulärer Dysfunktion}

Eine kardiale Dekompensation kann nicht nur durch eine Funktionseinschränkung des linken Ventrikels, sondern auch durch eine rechtsventrikuläre Dysfunktion bedingt sein. Die rechtsführende kardiale Dekompensation ist ebenfalls durch verminderter Belastbarkeit gekennzeichnet, jedoch stehen Beinödeme bzw. Anasarka und gestaute Halsvenen im Vordergrund, ggf. begleitet von akutem Nierenversagen und langfristig von der Entwicklung einer Cirrhose cardiaque mit Aszites. Der rechte Ventrikel ist weniger untersucht als der linke Ventrikel und wird aufgrund seiner komplexen halbmondförmigen Geometrie in der Routinediagnostik nur unzureichend evaluiert. Da sich die kardiovaskuläre Forschung bislang v.a. auf den linken Ventrikel fokussiert hat, gibt es in den aktuellen Herzinsuffizienzleitlinien keine spezifische Therapieempfehlung zur rechtsventrikulären
Dysfunktion. Es ist jedoch gut etabliert, dass eine zusätzliche rechtsventrikuläre Dysfunktion bei Linksherzinsuffizienz mit einer erhöhten Morbidität und Mortalität assoziiert ist $[8,9]$. Ein häufiger Grund für Rechtsherzinsuffizienz ist eine chronische Druckbelastung des rechten Ventrikels durch eine (sekundäre) pulmonale Hypertonie bei Linksherzinsuffizienz, teilweise mit der Entwicklung einer zusätzlichen präkapillären Komponente. Ziel bei rechtsventrikulärer Dysfunktion bei sekundärer pulmonaler Hypertonie ist daher eine Reduktion der Nachlast [10]. Bisher gibt es jedoch bei sekundär erhöhtem pulmonalvaskulärem Widerstand im Gegensatz zur primären pulmonalartiellen Hypertonie (WHO Klasse I) keine evidenzbasierte Therapie zur Senkung der Widerstände.

Grundsätzlich ist bei rechtsventrikulärer Dysfunktion und Dekompensation neben einer optimierten (Linksherzinsuffizienz-)Therapie ebenfalls eine Euvolämie anzustreben. Bei rechtsventrikulärer kardialer Dekompensation mit stationärer Aufnahme kann neben einer Intensivierung der diuretischen Therapie auch eine Therapie mit Milrinon oder die Inhalation mit dem Prostaglandin-Analogon Iloprost (Ilomedin ${ }^{\circledR}$ ) als „off-label use“ indiziert sein. Auch durch eine weitere Verschlechterung der linksventrikulä- ren Pumpfunktion kann es sekundär zu einem Rechtsherzversagen kommen.

Inwiefern durch PAP-Monitoring eine frühe Detektion bei führend rechtsventrikulärer Herzinsuffizienz und Dekompensation möglich ist, bleibt fraglich. Wie in - Abb. 4 ersichtlich, führten bei einem schwerst herzinsuffizienten Patienten die rezidivierenden rechtsführenden kardialen Dekompensationen zu keinen nennenswerten Veränderungen der PADruckkurven, sondern es kam jeweils zu starker Gewichtszunahme, Verschlechterung der Nierenfunktion und peripheren Ödemen mit konsekutiv erforderlicher Hospitalisierung. Ob es bei Rechtsherzversagen regelhaft nicht zu Veränderungen der nichtinvasiv gemessenen pulmonalarteriellen Druckwerte kommt oder ob es sich um einen Einzelfall handelt, muss in weiteren Studien untersucht werden.

\section{Dekompensation bei Vorhofflimmern}

Zusätzlich zu den pulmonalarteriellen Druckwerten wird bei jeder Übertragung auch die Frequenz der Pulsdruckkurven, entsprechend der effektiven Herzfrequenz, erfasst. Daher sollte bei jeder Prüfung der übermittelten Daten auch die Herzfrequenz überprüft werden, um die Sinnhaftigkeit weiterer Therapiemodifikationen sowie Arrhythmien 
wie tachykard übergeleitetes Vorhofflimmern oder vermehrte ventrikuläre Extrasystolie mit peripherem Pulsdefizit frühzeitig zu detektieren. Eine deutliche Abweichung der Herzfrequenz aus dem individuell festgelegten Korridor hinaus kann zu einer Verschlechterung der Herzinsuffizienz führen (• Abb. 5) und sollte daher einen Telefonkontakt mit dem Patienten nach sich ziehen.

\section{Nachsorge durch Heart Failure Nurses}

Qualifiziertes Personal, sog. Heart Failure Nurses, führen am Interdisziplinären Herzinsuffizienzzentrum strukturierte Telefoninterviews im Rahmen des Telemonitorings durch. Die Telefonkontakte werden bei stabilen Patienten in regelmäßigen zeitlichen Abständen durchgeführt:

- nach der ersten erfolgreichen Datenübertragung nach Entlassung,

- wöchentliche Telefonkontakte in den Wochen 1-4 nach Implantation,

- 2-wöchentliche Telefonkontakte in den Wochen 5-8 nach Implantation,

- 4-wöchentliche Telefonkontakte/E-

Mail-Kontakt ab der 9. Woche.

Bei den strukturierten Telefoninterviews wird zunächst nach dem aktuellen allgemeinen Befinden des Patienten gefragt und wie sich dies im Vergleich zu dem letzten Krankenhausaufenthalt bzw. dem letzten Telefonkontakt verändert hat. Hilfreich ist hierbei die Vergabe von Schulnoten von $1-5$, wobei 1 exzellent und 5 miserabel bedeutet. Hierbei können auch andere Erkrankungen wie eine Depression, die oft mit einer chronischen Herzinsuffizienz einhergehen, aufgedeckt und Behandlungsstrategien besprochen werden [11]. Danach erfolgt die Abfrage des Körpergewichts. Unsere Patienten werden angehalten, sich täglich zu wiegen. Bei kritischem Zustand kann auch eine zweimal tägliche (morgens und abends) Gewichtskontrolle sinnvoll sein. Wenn sich das Gewicht um > 1,5 kg über die letzte Woche verändert hat, wird die Diuretikadosierung entsprechend angepasst. Falls keine Gewichtskontrollen stattgefunden haben, wird der Patient erneut geschult und motiviert.
Anschließend wird der nichtinvasiv gemessene Blutdruck abgefragt. Sollte der Blutdruck systolisch $\geq 140 \mathrm{~mm} \mathrm{Hg}$ und/oder diastolisch $\geq 90 \mathrm{~mm} \mathrm{Hg}$ oder systolisch $\leq 85 \mathrm{mmHg}$ sein, so ist der Herzinsuffizienzarzt zu informieren und eine Therapieoptimierung $\mathrm{zu}$ diskutieren. Nun wird die Herzfrequenz eruiert. Sollte diese $>90 \mathrm{~min}^{-1}$ oder $<50 \mathrm{~min}^{-1}$ oder eine neu aufgetretene Irregularität aufweisen, ist ebenfalls der Herzinsuffizienzarzt zu informieren. Des Weiteren wird auf eine Dyspnoe/Fatigue-Symptomatik mit NYHA-Klassifikation und die Entwicklung oder Progredienz von Beinödemen eingegangen. Ebenso wichtig ist zu eruieren, ob sich kürzlich Stürze und/ oder Ohnmachtsanfälle ereignet haben. Anschließend werden Änderungen der Medikation abgefragt und notiert. Im Folgenden werden die Stimmungslage des Patienten, seine Compliance und die Belastbarkeit des Patienten im Alltag mit Schulnoten bewertet. Abschließend wird der nächste Telefonkontakt vereinbart, ungeklärte Fragen beantwortet und der Patient an das weitere Führen seines Herzinsuffizienztagebuchs (Blutdruck, Herzfrequenz, Gewicht, Blutzucker) erinnert.

Die Antworten des Patienten werden protokolliert, ebenso die durch den Herzinsuffizienzarzt getroffenen Anweisungen.

Bei Veränderungen des Zustands des Patienten wie Hypervolämie, Hypovolämie sowie nach Medikationsveränderungen und bei signifikanten Abweichungen der Trendwerte können auch häufigere Telefonkontakte (2-bis 3-mal/Woche) notwendig sein.

Sollte der PAP > $10 \mathrm{~mm} \mathrm{Hg} \mathrm{über} 2 \mathrm{Ta}-$ ge anhaltend ansteigen, ist der Patient so schnell wie möglich zu kontaktieren. Sollte der PAP-Wert $>15 \mathrm{~mm} \mathrm{Hg}$ ansteigen, muss der Patient sofort (auch an den Wochenenden und Feiertagen) kontaktiert werden, und es müssen Therapieoptimierungen erfolgen.

Aus den Rückmeldungen der Patienten wird deutlich, dass diese sich mit dem PAP-System sicherer fühlen, weil sie die Erfahrung gemacht haben, dass sie auch an Wochenenden, an Feiertagen und während Reisen kontaktiert werden, wenn Messungen auffällig sind.

\section{Bedeutung des Telemonitorings}

Mittels täglicher Übertragung von direkten Parametern der Hämodynamik stellt das Telemonitoring eine vielversprechende Option für schwer herzinsuffiziente Patienten dar. In kontrollierten klinischen Studien konnte bisher lediglich das CardioMEMS ${ }^{\mathrm{TM}}$-System eine signifikante Reduktion des primären und mehrerer sekundärer Endpunkte erreichen, welches nun auch in den aktuellen europäischen Herzinsuffizienz-Leitlinien als Klasse-IIb-Empfehlung seinen Niederschlag findet [12]. Hierbei ist zu betonen, dass nicht nur die zuverlässig funktionierende Technik, sondern v.a. die korrekte Nachsorge und angemessene zeitnahe Reaktion auf die gemessenen Werte ganz wesentlich für den Erfolg eines implantierbaren Hämodynamik-Telemonitoringsystems verantwortlich sind.

Aus gesundheitspolitischer Sicht ist es wichtig $\mathrm{zu}$ wissen, dass Anfang 2018 ein Potenzialbescheid für das CardioMEMS ${ }^{\mathrm{TM}}$-System durch das Institut für Qualität und Wirtschaftlichkeit im Gesundheitswesen (IQWiG) erfolgt ist, so dass einer durch den Gemeinsamen Bundesausschuss (GBA) in Auftrag gegebenen Studie zur Evaluation des Systems im deutschen Gesundheitssystem nichts mehr im Weg steht.

\section{Fazit für die Praxis}

Telemonitoring mittels täglicher
Übertragung von direkten Parame-
tern der Hämodynamik stellt eine
vielversprechende Option für schwer
herzinsuffiziente Patienten dar.
- Eine korrekte Nachsorge und ange-
messene zeitnahe Reaktion auf die
gemessenen Werte sind essenziell für
den Erfolg eines implantierbaren Hä-
modynamik-Telemonitoringsystems.
In Deutschland müssen hierfür neue
Strukturen und Personal (Heart Fail-
ure Nurses) implementiert werden,
um das ärztliche Personal nicht
zusätzlich zu binden.
Wichtig ist zudem die Entwicklung
eines Systems zur Qualitätskontrolle,
idealerweise in Kooperation mit
den Kostenträgern, um den Nutzen


und die korrekte Anwendung des hämodynamischen Monitorings langfristig zu etablieren.

\section{Korrespondenzadresse}

\section{Dr. med. Ester Herrmann}

Med. Klinik III, Kardiologie, Goethe Universität Frankfurt

Theodor-Stern-Kai 7, 60590 Frankfurt,

Deutschland

Ester.Herrmann@kgu.de

Danksagung. Die open access-Gebühren wurden von der Firma Abbott übernommen.

\section{Einhaltung ethischer Richtlinien}

Interessenkonflikt. E. Herrmann, A. Ecke, S. Fichtlscherer, A.M. Zeiher und B. Assmus geben an, dass kein Interessenkonflikt besteht.

Dieser Beitrag beinhaltet keine von den Autoren durchgeführten Studien an Menschen oder Tieren.

Open Access. Dieser Artikel wird unter der Creative Commons Namensnennung 4.0 International Lizenz (http://creativecommons.org/licenses/by/4.0/deed. de) veröffentlicht, welche die Nutzung, Vervielfältigung, Bearbeitung, Verbreitung und Wiedergabe in jeglichem Medium und Format erlaubt, sofern Sie den/die ursprünglichen Autor(en) und die Quelle ordnungsgemäßnennen, einen Link zur Creative Commons Lizenz beifügen und angeben, ob Änderungen vorgenommen wurden.

\section{Literatur}

1. Hendricks V et al (2014) Case management program for patients with chronic heart failure: effectiveness in terms of mortality, hospital admissions and costs. Dtsch Arztebl Int 111(15):264-270

2. Adamson PB (2009) Pathophysiology of the transition from chronic compensated and acute decompensated heart failure: new insights from continuous monitoring devices. Curr Heart Fail Rep 6(4):287-292

3. Rosenkranz S et al (2016) Left ventricular heart failure and pulmonary hypertensiondagger. Eur Heart J37(12):942-954

4. Abraham WT et al (2016) Sustained efficacy of pulmonary artery pressure to guide adjustment of chronic heart failure therapy: complete followup results from the CHAMPION randomised trial. Lancet 387(10017):453-461

5. Adamson PB et al (2011) CHAMPION trial rationale and design: the long-term safety and clinical efficacy of a wireless pulmonary artery pressure monitoring system. J Card Fail 17(1):3-10

6. Costanzo MR et al (2016) Interventions linked to decreased heart failure hospitalizations during ambulatory pulmonary artery pressure monitoring. JACC Heart Fail 4(5):333-344

7. Zile $M R$ et al (2017) Intracardiac pressures measured using an implantable hemodynamic monitor: relationship to mortality in patients with chronicheartfailure. Circ Heart Fail 10(1):e3594
8. Di Salvo TG et al (1995) Preserved right ventricular ejection fraction predicts exercise capacity and survival in advanced heart failure. J Am Coll Cardiol 25(5):1143-1153

9. Ghio $S$ et al (2001) Independent and additive prognostic value of right ventricular systolic function and pulmonary artery pressure in patients with chronic heart failure. J Am Coll Cardiol 37(1):183-188

10. Lin W, Poh AL, Tang WHW (2018) Novel insights and treatment strategies for right heart failure. Curr HeartFail Rep 15(3):141-155

11. Soucier RJ et al (2018) Essential elements of early post discharge care of patients with heart failure. Curr Heart Fail Rep 15(3):181-190

12. Ponikowski P et al (2016) 2016 ESC Guidelines for the diagnosis and treatment of acute and chronic heartfailure. Eur Heart J 37(27):2129-2200 